\title{
GENOMICS
}

\section{A genome for the environment}

Duplicated genes and many genes without known homologs might explain the enormous phenotypic adaptability of waterfleas.

\section{Dieter Ebert}

Universität Basel, Zoological Institute, Vesalgasse 1, 4059 Basel, Switzerland and Wissenschaftskolleg zu Berlin, Wallotstrasse 19, 14193 Berlin, Germany. Email: dieter.ebert@unibas.ch

Water fleas of the genus Daphnia are among the oldest model systems in biological research. Today, we know more about their natural history and ecology than of any other taxon. The Daphnia model also has left a notable mark on other fields. Élie Metchnikoff (1) used Daphnia to test his 1908 Nobel prizewinning idea that macrophages attack invading parasites as part of cellular immunity. August Weismann's studies on water fleas were instrumental in developing his theory that only germ cells transmit heritable information in animals (2). Richard Woltereck (3) used Daphnia to develop the notion of phenotypic plasticity-that an organism can change its characteristics in response to the environment-an idea that still guides experiments distinguishing genetic from environmental effects with many organisms. With all of these historical achievements, why did the National Institutes of Health (NIH) only recently add Daphnia to its list of model organisms (4) for biomedical research? Moreover, why has Daphnia, at this point in time, become NIH's 13th model system?

The answer to both questions lies, at least partly, in the very late start of genetic research on Daphnia. Until about 10 years ago, hardly any genetic experiments had been conducted on Daphnia. Even today, although researchers routinely cross clones, they have not yet developed methods for reverse genetics, including RNA interference. On page XXX of this issue, however, Colbourne et al. (5) take a substantial step forward in genetic research on the Daphnia system. They report on the genome of $D$. pulex - the world's first crustacean genomeand point to 37 companion papers (6). Their research demonstrates the powerful combination of genomic resources and ecological knowledge provided by the Daphnia system and opens the door to further exploration of the links between genomics and environmental adaptation.

Daphnia are small planktonic crustaceans ranging in length from less than 1 to about $5 \mathrm{~mm}$. Found all over the world in standing bodies of water, from small ephemeral pools to large lakes, Daphnia are keystone species in aquatic food 
chains. Their biology superbly suits them for scientific experimentation: They are transparent, have a short life cycle, and are easy and cost-effective to maintain through clonal reproduction. Daphnia clones can be crossed, which is important for genetic research. Lake sediments harbor layered archives of resting Daphnia eggs and sometimes even the transmission stages of the symbionts that have coeveolved with water fleas. Indeed, Daphnia and symbionts can be brought back to life and used to trace genetic adaptation over periods of environmental change (7).

Of the many intriguing features of Daphnia biology, the hallmark is their amazing phenotypic plasticity. Water fleas react to chemicals (kairomones) released by different predators by expressing highly specific characteristics, such as protective tail spines, helmets, and neck teeth (see the figure) (8). They are also able to adapt physiologically to wide ranges of $\mathrm{pH}$, toxins, oxygen concentrations, food, and temperature regimes (9). Moreover, maternal effects allow them to prepare their offspring for environmental challenges, including infectious disease (10), and the offspring's sex depends on the mother's environmental conditions. Colbourne et al. suggest that the secret of this success lies in the genome, with its unusually large repertoire of tandemly duplicated genes and a high proportion of genes specific to the Daphnia lineage.

Although the D. pulex genome is comparatively modest in size, measuring about 200 megabases, with more than 30,000 genes it exceeds the number many other genomes, including human genome. It also shares more genes with the human genome than any other arthropod model. However, $36 \%$ of D. pulex genes have no detectable homologs; many of them are part of lineage-specific gene families. About 13,000 Daphnia genes-an unusually high number-have been identified as paralogs, that is, genes within the same genome that are related by duplication. It appears that these genes arose not by whole genome duplications, as is the case for many human paralogs, but through an accumulative process by which the Daphnia genome gained more genes by tandem duplication and lost fewer genes than other lineages.

Gene duplication is an important evolutionary force $(11,12)$. The conventional view of the fate of duplicated genes is that genetic redundancy reduces constraints on their evolution, allowing for the accumulation of mutations. If these genes escape silencing, they may evolve altered functions. As a consequence, three variables - time since duplication, sequence divergence, and the degree of functional change-should correlate positively with each other, a pattern present in the Daphnia genome. In addition, Colbourne et al. support an entirely different model for the evolution of novel functions in duplicated genes. In this model, benefits arise from novel gene-gene interactions. The expression pattern of the duplicated gene changes as the result of interactions with a different interacting gene at, or soon after, the time of its duplication. Novel combinations of interacting genes may be expressed in a different tissue, phase of development, or under different environmental conditions. Novel expression 
patterns may arise by integrating the copied gene into a new genomic location or dissociating the gene from its previous regulatory framework. To support this claim, the authors provide evidence that paralogs, with no or little sequence differences, have in many cases diverged in their expression patterns. But where is the link to the environment? A strength of the Daphnia system, as the present study beautifully demonstrates, is the way it allows investigators to conduct gene expression experiments under diverse ecological conditions. Using expressed sequence tag (EST) libraries and genome-wide tiling microarray experiments, the authors demonstrate that genes responsive to specific ecological conditions are overrepresented in duplicated genes, in genes without known homologs, and in genomic regions without gene models (intergenic regions). Thus, $D$. pulex is equipped with a large array of genes with environment-specific functions, allowing these critters to call into play an extraordinary degree of phenotypic plasticity. The same seems to be true for $D$. magna, whose draft genome has been used in some of the comparative work conducted by Colbourne et al. (5).

The Daphnia model is currently being used in such fields as ecotoxicology, population genetics, the evolution of sex, phenotypic plasticity, ecophysiology (including global change biology), and Daphnia-parasite interactions. The publication of the $D$. pulex genome will allow this list to expand to embrace the emerging field of environmental genomics. Scientists in this field have already begun exploring how organisms adapt genetically to environmental stressors like heavy metals, rising temperatures, emerging diseases, and bioreactive organic compounds. By focusing on how organisms like Daphnia have evolved to cope with such stressors, this new approach goes well beyond questions of what these stressors are, and boldly takes on uncovering the kinds of solutions that evolution can bring about $(13,14)$. The signatures of these evolutionary answers, of course, are archived in the genomes of populations with different histories and ecologies. As global and local environmental change dominates world news today, understanding links between genes and environments becomes more and more vital. With the D. pulex genome, environmental health has found its genomic model.

\section{References and Notes}

1. M. E. Metchnikoff, Virchows Arch. Pathol. Anat. Physiol. 9, 177 (1884).

2. A. Weismann, Die Continuität des Keimplasma's als Grundlage einer

Theorie der Vererbung (Gustav Fischer, Jena, 1885).

3. R. Woltereck, Verh. Deutsch. Zool. Ges. 19, 110 (1909).

4. www.nih.gov/science/models.

5. J. K. Colbourne et al., Science 331, xxx (2011).

6. www.biomedcentral.com/series/.

7. $\quad$ E. Decaestecker et al., Nature 450, 870 (2007). 
8. C. Laforsch, W. Ngwa, W. Grill, R. Tollrian, Proc. Natl. Acad. Sci. U.S.A. 101, 15911 (2004).

9. S. Moenickes, O. Richter, R. Pirow, J. Exp. Biol. 213, 408 (2010).

10. F. Ben-Ami, D. Ebert, R. R. Regoes, Am. Nat. 175, 106 (2010).

11. S. Ohno, Evolution by Gene Duplication (Springer, New York, 1970).

12. M. Lynch, J. S. Connery, Science 290, 1151 (2000).

13. J. R. Shaw et al., BMC Genomics 8, 477 (2007).

14. R. Tollrian, F. Leese, BMC Biol. 8, 51 (2010).

15. C. Laforsch, R. Tollrian, Archiv Hydrobiol. 149, 587 (2000).

16. This perspective benefited from suggestions by L. Du Pasquier, M.

Pfrender, S. Zweizig, and N. R. Hunt.

Figure: Phenotypic plasticity. Members of the Daphnia genus, such as D. pulex (right pair) and $D$. longicephala (left pair), have the ability to alter their phenotypes in response to environmental cues. The presence of predators can induce the growth of neck teeth (inset), enlarged crests, and tail spines that reduce the animal's vulnerability to predation. The second and the third animal of are the uninduced forms.

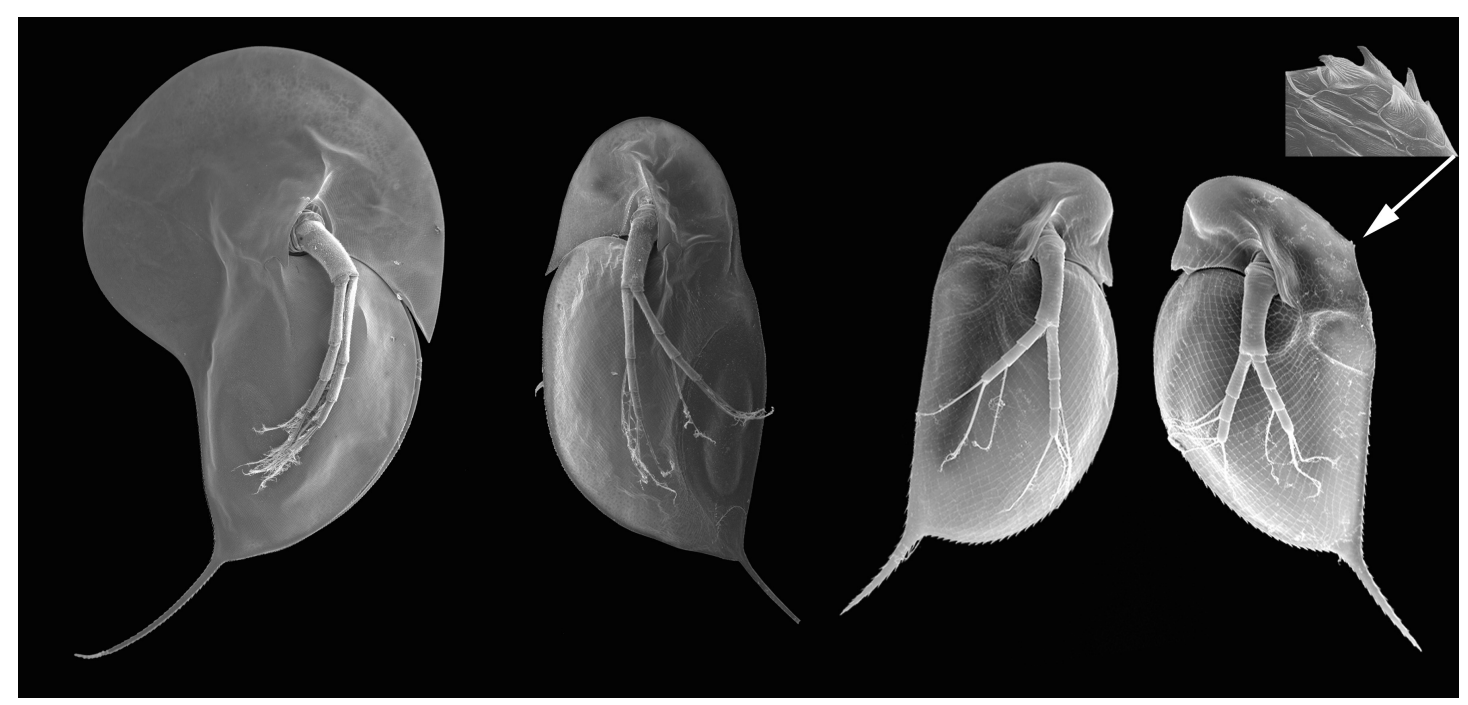

Credit: Scanning electron micrograph images (15) and copyright by C. Laforsch, LudwigMaximilians-Universität Munich, Germany. 